\title{
Migration mechanism of $<110\rangle$ tilt boundaries in nickel
}

\author{
G. M. Poletaev ${ }^{\dagger, 1}$, I. V. Zorya ${ }^{2}$, R. Y. Rakitin ${ }^{3}$ \\ †gmpoletaev@mail.ru
}

\author{
${ }^{1}$ Polzunov Altai State Technical University, 46 Lenina Av., Barnaul, 656038, Russia \\ ${ }^{2}$ Siberian State Industrial University, 25 Bardina Av., Novokuznetsk, 654007, Russia \\ ${ }^{3}$ Altai State University, 61 Lenina Av., Barnaul, 656049, Russia
}

\begin{abstract}
The features and migration mechanism of tilt boundaries with the misorientation axis $<110>$ in an fcc crystal using nickel as an example were studied by the method of molecular dynamics. The dependences of the boundaries energy and the rate of their migration at a temperature of $1700 \mathrm{~K}$ on the misorientation angle are obtained. It is shown that the migration rate of $<110>$ tilt boundaries under the same conditions is an order of magnitude lower than the migration rate of $<111>$ and $<100>$ boundaries, which is primarily due to the relatively low energy of $<110>$ boundaries. In addition, the low-angle $<110>$ tilt boundaries are unique compared to other tilt boundaries - grain boundary dislocations in them are ordinary perfect edge dislocations with straight cores that do not contain jogs periodically located on them, as in $<111>$ and $<100>$ boundaries. In $<110>$ boundaries, as well as in $<111>$ and $<100>$ boundaries, there are two different sets of dislocations, but they are not always combined, as is often the case in $<111>$ and $<100>$ boundaries. Combined dislocations in $<110>$ boundaries turned out to be less mobile during boundary migration than non-combined ones. An analogy of migration mechanisms of low-angle $<110>$ boundaries with the previously considered $<111>$ and $<100>$ boundaries was noted. During migration, in the grain towards which the migration took place, regions of the same shape orderly rotated through the angle of misorientation were formed, the size of which depended on the distance between neighboring grain boundary dislocations.
\end{abstract}

Keywords: molecular dynamics, grain boundary, migration, tilt boundary, migration mechanism.

\section{Introduction}

Grain boundary migration plays a decisive role in recrystallization process and in many instances of phase transformations. Despite the long-standing interest in the problem of migration of grain boundaries, there are still disagreements and unresolved issues. It is believed that low-angle tilt boundaries migrate through the combined action of two mechanisms: slip and climb of grain boundary dislocations [1]. In [2,3], for example, the authors come to the conclusion that the main mechanism of migration of the tilt boundaries is the climb of grain boundary dislocations. The cooperative climbing of dislocations likely took place during high-speed migration of symmetric boundaries, discovered by the authors of [4] in a computer model when shear stresses along the boundary were created in a sample. On the other hand, the results of $[5,6]$, on the contrary, indirectly support the predominance of the slip mechanism of grain boundary dislocations.

Previously in $[7,8]$, we have studied the atomic mechanism of migration of tilt boundaries with $<100>$ and $<111>$ misorientation axes, as well as their triple junctions, using the molecular dynamics method. It was shown that the migration and elongation of the low-angle $<100>$ boundaries are occurred by splitting of the paired grain boundary dislocations with the subsequent change of the dislocationspartners. The dislocations-partners are replaced through the slip of split dislocations. The migration of the $<111>$ tilt boundaries occurred as a result of the combined action of two mechanisms: the mechanism described above and the mechanism consisting in the joint slipping of paired grain boundary dislocations, which, in contrast to grain boundary dislocations in the $<100>$ boundaries, have common slip planes.

In our previous works, we did not consider the boundaries with the misorientation axis $<110\rangle$. However, the consideration of $\langle 110\rangle\{111\}$ tilt boundaries is of great practical importance, since, as is known, grain boundaries oriented in the most closely packed $\{111\}$ planes are more common in fcc metals $[9,10]$, and among tilt boundaries are more common with the misorientation axis $<110>[9-13]$. This type of boundaries, for example, also includes twins $\Sigma 3\{111\}<110>[14,15]$.

The present work is devoted to the study of the peculiarities and migration mechanism of low-angle $<110>$ tilt boundaries using the example of nickel by the molecular dynamics method.

\section{Description of the model}

The model in the present work is based on the technique for studying the migration of tilt grain boundaries, proposed and developed in $[1,16]$. A clearly defined boundary was created in the form of a half-loop, as in Fig. 1 (black dotted line). The tension of the boundary in this case, which, like surface tension, arises from the tendency of the boundary to 


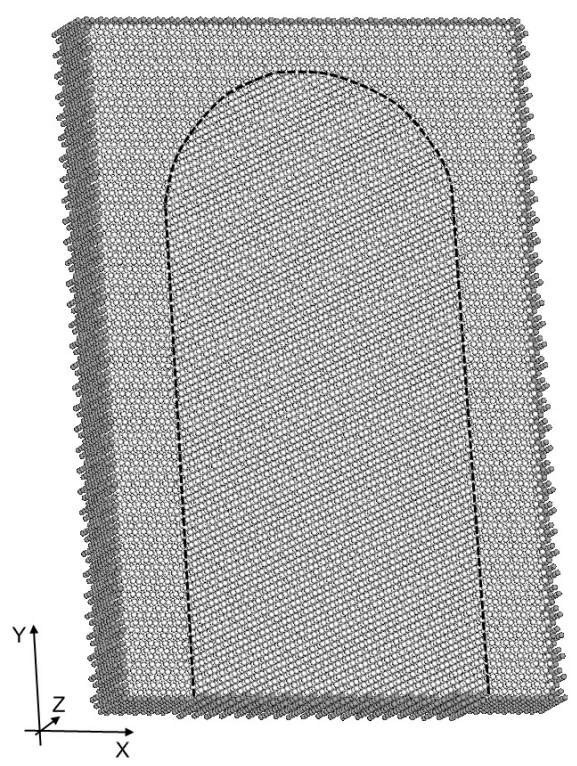

Fig. 1. Computational cell for modeling the migration of $<110>30^{\circ}$ tilt boundary. The dark-gray atoms on the edge of the computational cell remained motionless during the computer experiment (rigid boundary conditions).

minimize its energy, is the reason for the directed movement of the boundary at the top part of the "half-loop" toward a decrease in its area. The force that provokes the boundary migration depends on the misorientation angle (as a rule, due to an increase in the boundary energy with an increase in the misorientation angle) (Fig. 2) and the boundary curvature. In our model, a relatively high boundary curvature was chosen so that the migration rate was high enough to be measured in the molecular dynamics model. The force and migration rate at the same conditions (temperature, misorientation angle, computational cell size and boundary curvature) remains almost constant throughout almost the entire movement of the boundary, smoothly decreasing by the end of the computer experiment.

The computational cells in the molecular dynamics model contained approximately 60000 atoms and had a height of $20.1 \mathrm{~nm}$, a width of $13.6 \mathrm{~nm}$, and a thickness of $2.5 \mathrm{~nm}$ (Fig. 1). Along the Z-axis, an infinite repetition of the

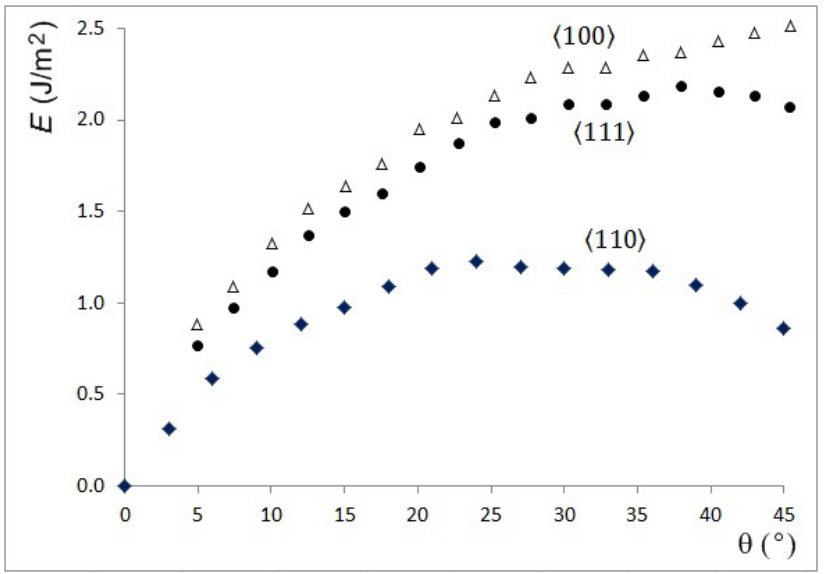

a structure was simulated, i. e. the periodic boundary conditions were imposed. At the edge of the computational cell, the grain boundaries must be fixed, which implies preserving the orientation of the crystal lattice of two different grains at the border of the cell. In this connection, along the $\mathrm{X}$ and $\mathrm{Y}$ axes, the cell borders (highlighted in dark gray in Fig. 1) were rigidly fixed to preserve the given misorientation of the grains.

Interactions between atoms in the computer model were described by the many-body Cleri-Rosato potential, built within the tight-binding model [17]. Potentials of this type had been used repeatedly in molecular dynamics models and tested for a large number of characteristics $[18,19]$. These applications proved their possible availability to describe well various properties of metals and alloys.

Simulation of the migration of grain boundaries was carried out at a temperature of $1700 \mathrm{~K}$. The choice of a temperature almost equal to the melting point is due to the fact that at this temperature the highest rate of grain boundary migration is observed. The temperature in the model was set through the initial velocities of the atoms according to the Maxwell-Boltzmann distribution, wherein the thermal expansion of the calculation blocks was taken into account. To keep the temperature constant during the simulation, the Nose-Hoover thermostat was used.

\section{Results and discussion}

Fig. 2 shows the dependences of $<110>$ tilt boundary energy and the rate of their migration at a temperature of $1700 \mathrm{~K}$ on the misorientation angle. For comparison, the corresponding values for $<111>$ and $<100>$ boundaries from [8] are given. Boundaries with a misorientation angle from $0^{\circ}$ to $45^{\circ}$ were considered. The grain boundary energy was calculated as the ratio of the energy difference between the computational cell with the boundary and the same number of atoms in an ideal crystal to the boundary area. Before calculating the energy, the structure was relaxed. The obtained energy values are in good agreement with the results of other authors $[11,12]$.

At the considered temperature of $1700 \mathrm{~K}$, which is close to the melting point of nickel, the migration of $\langle 110\rangle$ boundaries with a misorientation angle above $10^{\circ}$ occurred

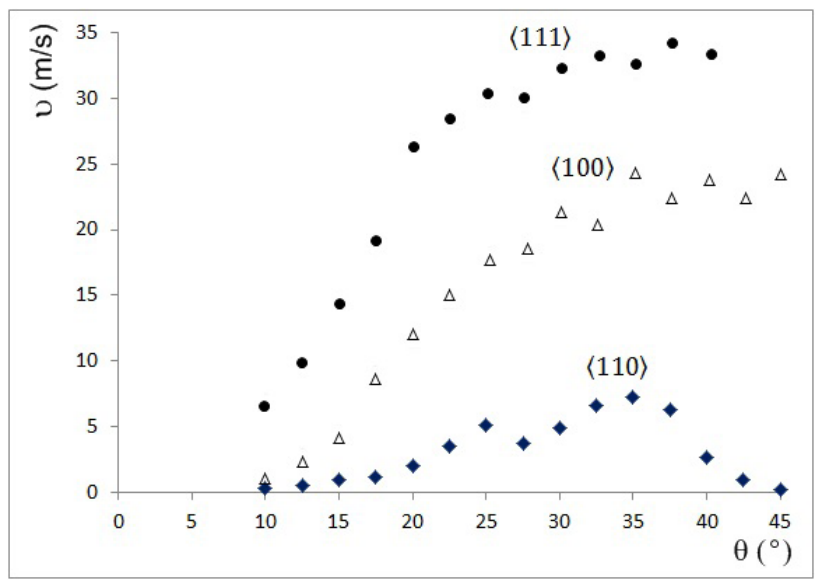

b

Fig. 2. Energy of $\langle 110\rangle,\langle 111\rangle$, and $\langle 100\rangle$ tilt boundaries (a) and their migration rate (b) at a temperature of $1700 \mathrm{~K}$ depending on the misorientation angle $\theta$ in $\mathrm{Ni}$. 
at a sufficiently high rate to be measured in the molecular dynamics model. The migration rate during the simulation remained approximately constant, which made it relatively easy to determine it as the ratio of the displacement of the upper part of the boundary (Fig. 1) to the time of the molecular dynamics experiment.

Low-angle $<110>$ tilt boundaries are unique compared to other tilt boundaries - grain boundary dislocations at such boundaries are ordinary perfect edge dislocations with straight cores that do not contain jogs (or steps) on them. At the tilt boundaries with other misorientation axes, for example $<111>$ and $<100>$, grain boundary dislocations are more complex, they are, as a rule, paired (i. e. dislocations from two sets with different slip planes combined in one core) and contain obligatory jogs along the cores $[7,8]$. Dislocations in $<110>$ tilt boundaries do not have jogs and are relatively very mobile, which makes them unique. $<110>$ boundaries have a relatively low diffusion permeability and high diffusion anisotropy even in the case of high-angle boundaries [20]. In this regard, most likely, they contain less free volume, and their energy is significantly lower than the energy of $<111>$ and $\langle 100\rangle$ boundaries at the same misorientation angles (Fig. 2 a).

Despite the relatively high mobility of dislocations in low-angle $<110>$ boundaries, their migration proceeded almost an order of magnitude slower than $<111>$ and $<100>$ boundaries [8], which is obviously associated with the relatively low energy of $\langle 110\rangle$ boundaries. At the same conditions, temperature and size of the computational cell, the migration rate of $\langle 111\rangle$ and $\langle 100\rangle$ boundaries with a misorientation angle above $30^{\circ}$ was in [8] about $30-35 \mathrm{~m} / \mathrm{s}$. The peak of the migration rate of $\langle 110\rangle$ boundaries was observed for high-angle boundaries from $22^{\circ}$ to $37^{\circ}$ (Fig. 2 b). Then the rate decreased again, dropping to almost zero at a misorientation angle of $45^{\circ}$.

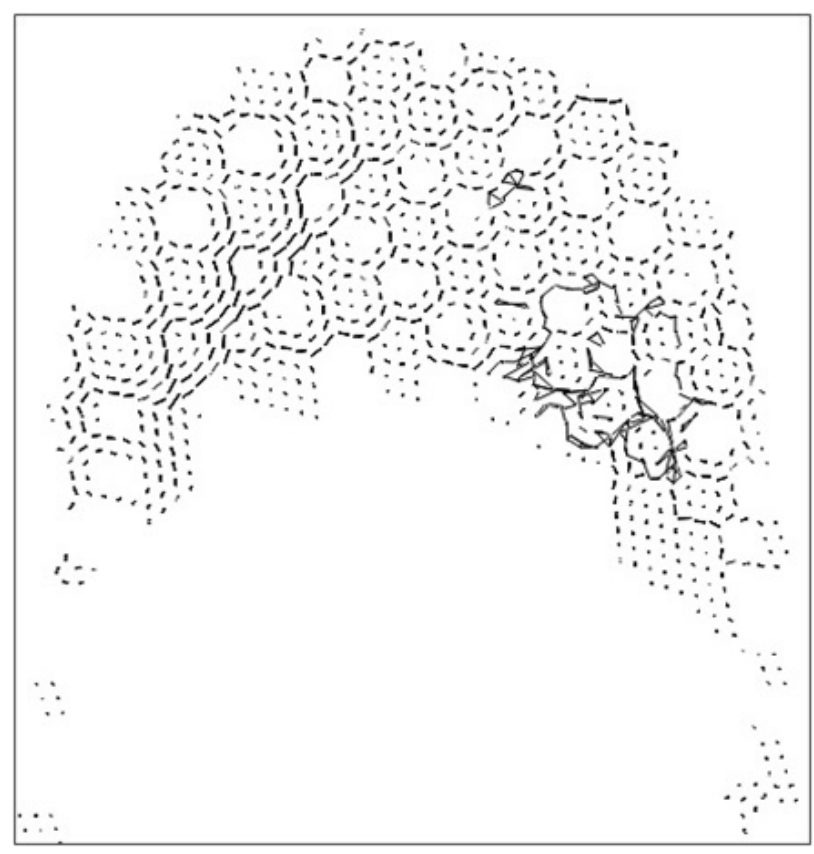

a
Fig. 3 shows examples of atomic displacements during migration of $\langle 110\rangle$ boundaries with misorientation angles of $15^{\circ}$ and $25^{\circ}$. Despite the fact that boundaries with a misorientation angle of $25^{\circ}$ are already referred to as highangle boundaries, the trajectories of atomic displacements during migration of $15^{\circ}$ and $25^{\circ}$ boundaries form similar "grids" (Fig. $3 \mathrm{a}$ and $3 \mathrm{~b}$ ). The analogy of the migration mechanisms of $\langle 110\rangle$ boundaries with the mechanisms of migration of $\langle 111\rangle$ and $\langle 100\rangle$ boundaries considered earlier in $[7,8]$ is clearly visible. During migration, in the grain towards which the migration took place, regions of the same shape orderly rotated through the angle of misorientation were formed, the size of which, in the case of low-angle boundaries, depended on the distance between neighboring grain boundary dislocations. The shape of these areas is determined by crystallography. In the case of $<110>$ boundaries, they almost have hexagonal shape.

Low-angle $<110>$ boundaries, in contrast to $<111>$ and $<100>$ boundaries, much less often contain paired (combined) dislocations. As mentioned above, grain boundary dislocations in $<110\rangle$ boundaries are ordinary perfect edge dislocations, but with a very short stacking fault between partial dislocations (Fig. 4). In $<110>$ boundaries, as well as in $\langle 111\rangle$ and $<100\rangle$ boundaries, there are two different sets of dislocations (in Fig. 4, stacking faults between partial dislocations of two different sets of grain boundary dislocations are shown in different colors), but they are not always combined, as is often the case in $<111>$ and $<100>$ boundaries $[7,8]$. Such combined dislocations, as can be seen in Fig. 4 , in $<110>$ boundaries turned out to be less mobile during boundary migration than non-combined ones. Basically, migration proceeded with the participation of single dislocations. In the given example (Fig. 4), they slid downward, but then a consistent shift took place (the most intense atomic displacements in the horizontal

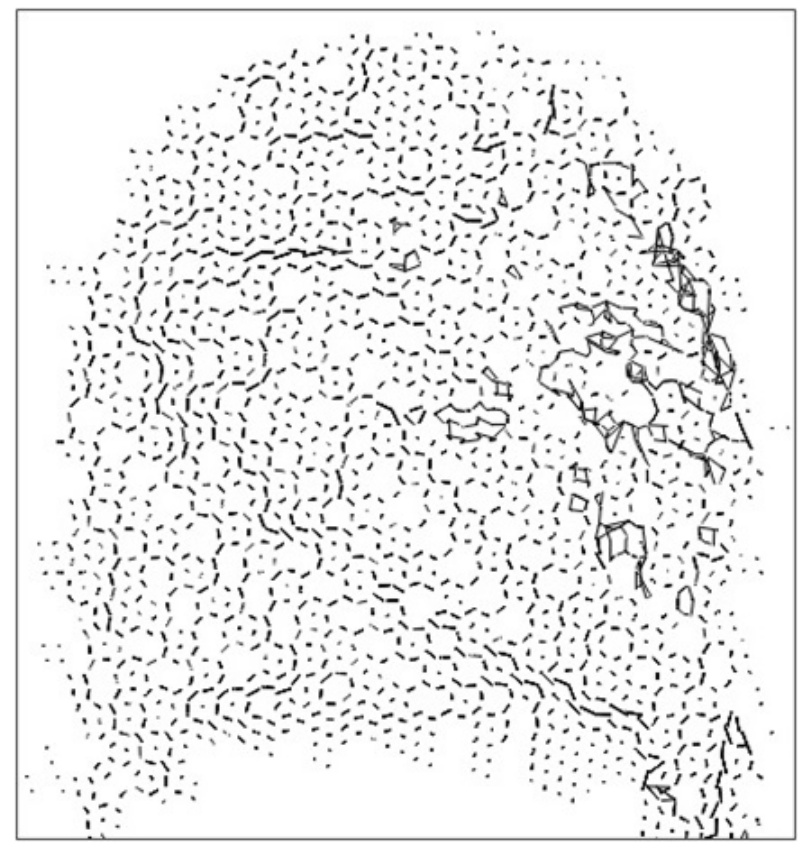

$\mathrm{b}$

Fig. 3. Atomic displacements (more than $0.08 \mathrm{~nm}$ ) in the process of migration of $<110>15^{\circ}$ (during $3000 \mathrm{ps}$ ) (a) and $<110>25^{\circ}$ (during 1500 ps) (b) tilt boundaries in Ni at $1700 \mathrm{~K}$. 


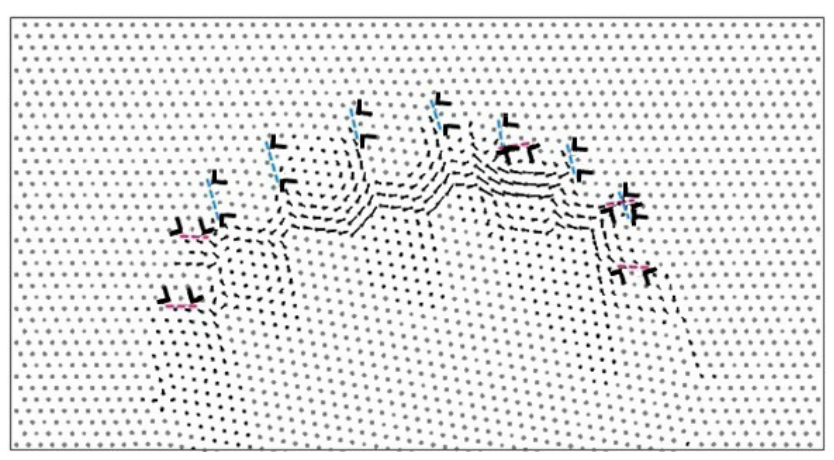

Fig. 4. Atomic displacements in the process of migration of $\langle 110\rangle$ $10^{\circ}$ tilt boundary (during $4000 \mathrm{ps}$ ) with indication of the initial position of grain boundary dislocations.

direction), leading to a rotation of the hexagonal regions by the misorientation angle. This consistent shift is obviously cooperative and does not contain diffusion elements.

It should be noted that the character of atomic displacements in $\langle 110\rangle$ grain boundaries is also the same as for $<111>$ and $<100>$ boundaries $[7,8]$. In all cases, migration was occurred through cooperative shears as a result of coordinated sliding of grain boundary dislocations from different sets. The dislocation climbing caused by diffusion, according to our studies, practically did not contribute to the mechanism of boundary migration.

\section{Conclusion}

The molecular dynamics method was used to study the structural features and migration mechanism of tilt boundaries with the misorientation axis $\langle 110\rangle$ in an fcc crystal using nickel as an example. The dependences of the considered boundaries energy and the rate of their migration at a temperature of $1700 \mathrm{~K}$ on the angle of misorientation are obtained. It is shown that the migration rate of $\langle 110\rangle$ tilt boundaries under the same conditions is an order of magnitude lower than the migration rate of $\langle 111\rangle$ and $<100>$ boundaries, which is primarily due to the relatively low energy of $\langle 110\rangle$ boundaries. In addition, the lowangle $\langle 110\rangle$ tilt boundaries are unique compared to other tilt boundaries - grain boundary dislocations in them are ordinary perfect edge dislocations with straight cores that do not contain jogs periodically located on them, as, for example, in the $\langle 111\rangle$ and $\langle 100\rangle$ boundaries. In $<110\rangle$ boundaries, as well as in $\langle 111\rangle$ and $\langle 100\rangle$ boundaries, there are two different sets of dislocations, but they are not always combined, as is often the case in $\langle 111\rangle$ and $\langle 100\rangle$ boundaries. Combined dislocations in $\langle 110\rangle$ boundaries turned out to be less mobile during boundary migration than non-combined ones.

An analogy of migration mechanisms of low-angle $<110>$ boundaries with the previously considered $<111>$ and $<100>$ boundaries was noted. During migration, in the grain towards which the migration took place, regions of the same shape orderly rotated through the angle of misorientation were formed, the size of which, in the case of low-angle boundaries, depended on the distance between neighboring grain boundary dislocations. The migration of all considered $<111>,<100\rangle$, and $<110>$ low-angle boundaries was occurred through cooperative shears as a result of coordinated sliding of grain boundary dislocations from different sets. The diffusional dislocation climbing, according to our studies, practically did not contribute to the mechanism of boundary migration.

\section{References}

1. G. Gottstein, L.S. Shvindlerman. Grain Boundary Migration in Metals: Thermodynamics, Kinetics, Applications. 2nd ed. Boca Raton, CRC Press (2009) 711 p. Crossref

2. R.W. Balluffi, J.W. Cahn. Acta Metallurgica. 29, 493 (1981). Crossref

3. M. Winning, A. D. Rollett, G. Gottstein, et al. Philosophical Magazine. 90, 3107 (2010). Crossref

4. K.P. Zolnikov, D.S. Kryzhevich, A. V. Korchuganov. Letters on Materials. 9 (2), 197 (2019). $\underline{\text { Crossref }}$

5. Y. Huang, F. J. Humphreys. Acta Materialia. 47, 2259 (1999). Crossref

6. Y. Huang, F. J. Humphreys. Materials Chemistry and Physics. 132, 166 (2012). Crossref

7. G. Poletaev, I. Zorya, R. Rakitin. Computational Materials Science. 148, 184 (2018). Crossref

8. G. M. Poletaev, I.V. Zorya, M.D. Starostenkov, et al. Journal of Experimental and Theoretical Physics. 128 (1), 88 (2019). $\underline{\text { Crossref }}$

9. J. Li, S. J. Dillon, G. S. Rohrer. Acta Materialia. 57, 4304 (2009). Crossref

10. S. Ratanaphan, D.L. Olmsted, V. V. Bulatov, et al. Acta Materialia. 88, 346 (2015). Crossref

11. D. L. Olmsted, S. M. Foiles, E. A. Holm. Acta Materialia. 57, 3694 (2009). Crossref

12. V. V. Bulatov, B. W. Reed, M. Kumar. Acta Materialia. 65, 161 (2014). Crossref

13. M.A. Tschopp, Sh.P. Coleman, D.L. McDowell. Integrating Materials and Manufacturing Innovation. 4, 11 (2015). Crossref

14. N. V. Malyar, B. Grabowski, G. Dehm, et al. Acta Materialia. 161, 412 (2018). Crossref

15. Y. Liang, X. Yang, M. Gong, et al. Computational Materials Science. 161, 371 (2019). Crossref

16. S. G. Protasova, V.G. Sursaeva, L.S. Shvindlerman. Physics of the Solid State. 45, 1471 (2003). Crossref

17. F. Cleri, V. Rosato. Physical Review B. 48 (1), 22 (1993). Crossref

18. G. M. Poletaev, D. V. Novoselova, I. V. Zorya, et al. Physics of the Solid State. 60 (5), 847 (2018). Crossref

19. G. M. Poletaev, I. V. Zorya. Technical Physics Letters. 46 (6), 575 (2020). Crossref

20. I. V. Zorya, G. M. Poletaev, M.D. Starostenkov. Fundamentalnye problemy sovremennogo materialovedenia. 17 (1), 45 (2020). (in Russian) 